\title{
Rosette-forming epithelioid osteosarcoma in the rib: a rare case of location and morphology
}

\author{
Sun-Ju Oh \\ Department of Pathology, Kosin University College of Medicine, Busan, Korea
}

\begin{abstract}
The rib is an unusual location for osteosarcoma and is reported in only $2 \%$ of all cases. The major histological variants of osteosarcoma are osteoblastic, chondroblastic, and fibroblastic, with a few rare variants including one epithelioid type. This report describes a 44-yearold male with an osteolytic mass in the right seventh rib. Histological examination revealed osteosarcoma with unique features of epithelioid appearance and rosette structures. To the best of our knowledge, this is the first reported case of a rosette-forming osteosarcoma of the rib that showed epithelioid morphology. Despite successful surgery, the patient's prognosis was poor because this malignancy had an unusual location within the axial skeleton and was a rare histological variant.
\end{abstract}

Key Words: Osteosarcoma; Ribs; Epithelioid; Rosette

Received: May 7, 2021 Revised: May 25, 2021 Accepted: June 22, 2021

Corresponding Author: Sun-Ju Oh, Department of Pathology, Kosin University College of Medicine, 262 Gamcheon-ro, Seo-gu, Busan 49267, Korea

Tel: +82-51-990-6333, Fax: +82-51-990-3080, E-mail: 10highpowerfield@gmail.com

Osteosarcoma (OSA) is the most common primary malignant bone tumor, and it typically occurs in adolescents and young adults, with a slight male predominance [1]. OSA primarily affects the metaphysis of the long bones and, less frequently, the axial skeleton, including the ribs [2]. Various pathological subtypes of conventional OSA have been recognized, including osteoblastic, chondroblastic, and fibroblastic subtypes. This report describes a rare case of epithelioid OSA that involved the rib with special rosette-forming structure morphological features. Particular attention was paid to review the OSA in the rib, which is an atypical location for a lesion, and the rare histologic rosetteforming epithelioid OSA variant.

\section{CASE REPORT}

A 44-year-old male presented with a mass in the right posterior thorax. He reported intermittent upper back pain for the 6 months prior to admission. An initial chest radiograph showed a nonhomogeneous mass that involved the posterolateral aspect of the right 7 th rib, and contrast-enhanced chest computed tomography $(\mathrm{CT})$ indicated an ill-defined osteolytic lesion with coarse calcification (Fig. 1). Overall image analysis suggested a malig- nant bone tumor, including chondrosarcoma. The patient underwent wide surgical excision of the rib with tumor-free margins.

Gross examination revealed resected bone with a 6-cm-sized hemorrhagic mass with cortical disruption (Fig. 2A). Microscopically, the tumor was arranged in a trabecular or rosette-like growth pattern that contained a fibrillar matrix in the center (Fig. 2B, C). Higher magnification exhibited epithelioid tumor cells with a plasmacytoid feature of eccentrically located nuclei and an abundant cytoplasm (Fig. 2C, D). The nuclei showed a clumped chromatin pattern, and each contained 1 or 2 prominent nucleoli. Mitoses were frequent, up to 12 per 10 high-power fields. An area of well-defined nests was identified, whereas blood-filled cystic spaces exhibited telangiectatic features (Fig. 2E). An irregularly interconnected coarse trabecular pattern of immature osteoid deposition was obvious throughout the tumor (Fig. 2F). For immunohistochemical panels, the neoplastic cells stained negatively for cytokeratin AE1/AE3, leukocyte common antigen, CD138, CD99, synaptophysin, desmin, and CD34, with the only exception of a positive reaction for vimentin. The final histologic diagnosis was a rosette-forming epithelioid osteosarcoma.

The postoperative systemic workup did not reveal metastasis, and the patient was treated with adjuvant radiotherapy for pro- 

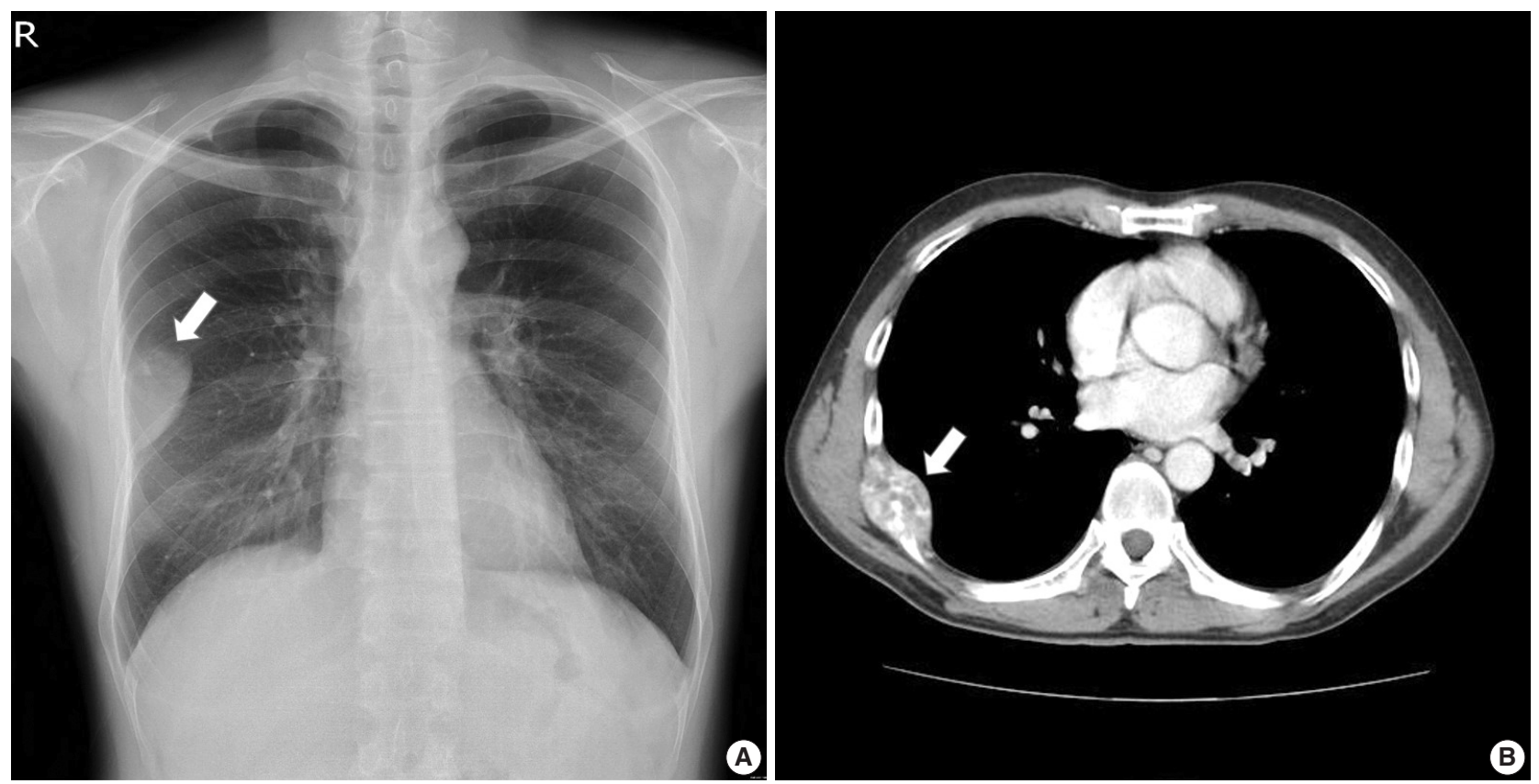

Fig. 1. Radiologic images. (A) Chest radiograph showing an oval mass (arrow) in the right thorax. (B) Computed tomography scan of the chest, revealing a large, heterogeneously enhanced mass (arrow) that originated from the right seventh rib, with lytic destruction of the bone and coarse calcification.
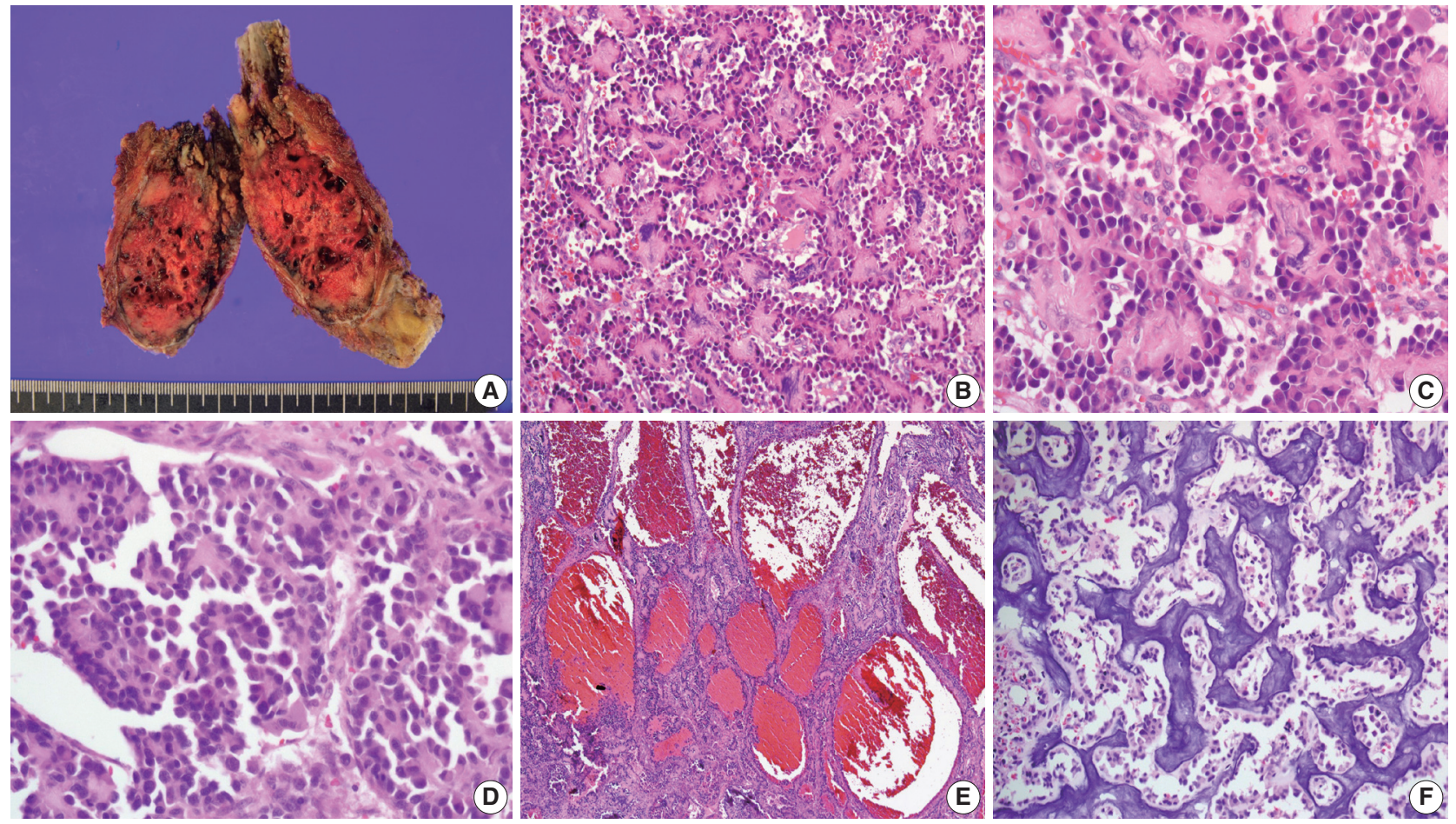

Fig. 2. Epithelioid osteosarcoma of the rib. (A) Macroscopic picture of an ill-defined lytic mass of the rib with hemorrhagic cystic degeneration. (B) Microscopic view of the trabecular and rosette-forming pattern that contained a fibrillar matrix in the center. (C) Rosette-like structures of plasmacyoid tumor cells with eccentrically located nuclei and abundant pale cytoplasm. (D) Mixed epithelioid and plasmacytoid tumor cells. (E) Blood-filled cystic spaces showing telangiectatic features. (F) Neoplastic bones produced by malignant tumor cells are found throughout the lesion. 
phylactic purposes. After one year of follow-up, however, recurrence was noted on the adjacent ribs and chest wall, for which the patient underwent palliative surgery followed by six cycles of adjuvant chemotherapy that consisted of doxorubicin and cisplatin. Tumor progression continued with multiple metastases to both lungs and the liver, and the patient died four and a half years after initial diagnosis.

\section{DISCUSSION}

Primary OSA of the ribs is uncommon, and accounting for only 40 (2\%) of the total 1,952 cases of OSA reported in the largest series; to date, very few cases have been reported [1-5]. Most of the cases that affect the ribs occurred in patients during their fourth decade, which was late compared to cases of the long bone, which primarily occur in the second decade. The major histological variants of conventional OSA are subdivided as osteoblastic, chondroblastic, and fibroblastic, while a few other variants are classified as giant cell-rich, osteoblastoma-like, and clear cell [1]. Scranton et al. first reported the rare epithelioid OSA subtype [6]. Several subsequent reports have described cases of epithelioid OSA that affect long bones and the lumbar spine, maxilla, and mandible [7-10]. Only one epithelioid OSA that originated in the ribs was reported prior to this case [11]. In one of these epithelioid cases, tumor cells showed various patterns of sheet, alveolar, acinar, trabecular, organoid, hemangiopericytoma-like, and rosette-like appearances [9]. Rosette-forming OSA was illustrated in only two previous studies [8,9]. In the two studies, Okada et al. found a rosette-like structure in 16 of 280 cases of OSA, which corresponded with a rate of $5.7 \%$ [9].

Histopathological differential diagnosis in epithelioid tumors of the rib are comprised of various malignant neoplasms including metastatic carcinoma, malignant melanoma, and any kind of malignant mesenchymal tumor with an epithelioid appearance such as a fibrosarcoma, leiomyosarcoma, and an undifferentiated sarcoma. Although immunohistochemistry is helpful for differentiation, the tissue samples must be carefully examined to identify malignant osteoid formation by tumor cells, which is pathognomonic for OSA. Metaplastic bone formations and calcifications are relatively common in many benign and malignant tumors, but only lace-like osteoid formation by malignant cells is important in the differential diagnosis.

Reported outcomes of patients with OSA indicated traumatic improvements after wide excision and multi-agent chemotherapy [1]. This strategy of extensive surgical resection and adjuvant chemotherapy are also effective in OSA of the ribs and increased the likelihood of relapse-free survival [2]. Another study, however, indicated that there was no apparent difference in survival rates between patients who underwent surgery alone and those who underwent both surgery and chemotherapy [4]. Surgical resection should include the entire thickness of the chest wall with a wide margin, including the adjacent ribs, intercostal muscles, pleura, and vertebrae. After resection, large chest wall defects need to be covered with tissue flaps or mesh material [4].

The prognosis of OSA in the axial skeleton, including the rib, is generally poorer than results reported for cases in the extremity [1]. The 5-year survival rate for patients with rib OSA was much lower, at $27 \%$, despite multimodal chemotherapy and aggressive surgery, compared to the higher $65 \%-75 \%$ survival rate in patients with OSA in an extremity [4]. Okada et al. [9] reported that rosette-forming OSA is more aggressive than other histological variants; the 5 -year survival rate was $15 \%$ compared to $45 \%$ for OSA without rosettes. In addition, $75 \%$ of patients with rosette characteristics died of multiple lung metastases despite extensive surgery with wide surgical margins and systemic chemotherapy [9].

In conclusion, this is the first report of a rosette-forming epithelioid OSA that involved the rib. This case illustrates the unusual morphological features. Because this OSA is a rare subtype and associated with a poor prognosis, additional information regarding proper treatment is needed.

\section{Ethics Statement}

This study was approved by the institutional review board (IRB) of Kosin University Gospel Hospital with a waiver of informed consent (IRB No. 2018-01-020).

\section{Availability of Data and Material}

The datasets generated or analyzed during the study are available from the corresponding author on reasonable request.

\section{Code Availability}

Not applicable.

\section{ORCID}

Sun-Ju Oh

$$
\text { https://orcid.org/0000-0001-6013-8579 }
$$

\section{Conflicts of Interest}

The authors declare that they have no potential conflicts of interest.

\section{Funding Statement}

No funding to declare.

\section{References}

1. Unni KK, Inwards CY. Dahlin's bone tumors. 6th ed. Philadelphia: Lippincott Williams \& Wilkins, 2010; 122-57. 
2. Chattopadhyay A, Nagendhar Y, Kumar V. Osteosarcoma of the rib. Indian J Pediatr 2004; 71: 543-4.

3. Ikeda H, Takeo M, Kayata H, Mikami R, Nakamoto Y, Yamamoto M. A case of rapidly growing osteosarcoma of the rib. Ann Thorac Cardiovasc Surg 2014; 20 Suppl: 521-4.

4. Deitch J, Crawford AH, Choudhury S. Osteogenic sarcoma of the rib: a case presentation and literature review. Spine (Phila Pa 1976) 2003; 28: E74-7.

5. Mohanty S, Inchara YK, Crasta JA, Ananthamurthy A. An unusual case of primary osteosarcoma of the rib in an adult. Indian J Med Paediatr Oncol 2010; 31: 18-20.

6. Scranton PE Jr, DeCicco FA, Totten RS, Yunis EJ. Prognostic factors in osteosarcoma: a review of 20 year's experience at the University of Pittsburgh Health Center Hospitals. Cancer 1975; 36: 2179-91.

7. Kramer K, Hicks DG, Palis J, et al. Epithelioid osteosarcoma of bone: immunocytochemical evidence suggesting divergent epitheli- al and mesenchymal differentiation in a primary osseous neoplasm. Cancer 1993; 71: 2977-82.

8. Carlos-Bregni R, Contreras E, Hiraki KR, Vargas PA, Leon JE, de Almeida OP. Epithelioid osteosarcoma of the mandible: a rare case with unusual immunoprofile. Oral Surg Oral Med Oral Pathol Oral Radiol Endod 2008; 105: e47-52.

9. Okada K, Hasegawa T, Yokoyama R. Rosette-forming epithelioid osteosarcoma: a histologic subtype with highly aggressive clinical behavior. Hum Pathol 2001; 32: 726-33.

10. Kaveri H, Rekha K, Punnya VA. Epithelioid osteosarcoma of the maxilla: report of an unusual case. Br J Oral Maxillofac Surg 2009; 47: 143-5.

11. Kuwabara H, Fujita K, Yuki M, Goto I, Hanafusa T, Shibayama Y. Cytokeratin-positive rib osteosarcoma metastasizing to the small intestine. Indian J Pathol Microbiol 2014; 57: 109-12. 